\title{
WHAT LIES BETWEEN RIGIDITY AND FLEXIBILITY OF STRUCTURES
}

A B S T R A C T

The borderline between continuous flexibility and rigidity of structures like polyhedra or frameworks is not strict. There can be different levels of infinitesimal flexibility. This article presents the mathematical background and some examples of structures which under particular conditions are flexible or almost flexible and otherwise rigid. 


\section{INTRODUCTION}

A framework or a polyhedron will be called "rigid" when the edge lengths determine its planar or spatial shape uniquely; under the term "shape" we mean its spatial form - apart from movements in space.

More generally and under inclusion of smooth or piecewise linear surfaces, a structure is called rigid when its intrinsic metric defines its spatial shape uniquely. In this sense, the intrinsic metric of a polyhedron is defined by its net (unfolding), i.e., the coplanar set of faces with identified pairs of edges originating from the same edge of the spatial form. After cutting out this net from paper or cardboard, a paper- or cardboard-model of this polyhedron can be built in the usual way. Does such a net really define the shape of a polyhedron uniquely?

Think of a cube where one face is replaced by a four-sided pyramid with a small height. Then, obviously two different polyhedra can be built, one convex form with the pyramid erected towards outside, the other with the apex of the pyramid inside the cube (Fig. 1). So, there are two polyhedra, two realizations, stemming from the same net, i.e., with the same intrinsic metric. In the convex case the internal dihedral angles along the edges of the pyramid are $<180^{\circ}$, in the other case they are $>180$.

If the height is sufficiently small, distort the convex polyhedron can be distorted by applying slight force and change to the concave form. In this case we speak of "snapping" polyhedra. Both realizations are "locally rigid", i.e., there is no other realization of the same intrinsic metric sufficiently close to the one under consideration. "Globally rigid" is a structure for which the intrinsic metric defines its spatial shape uniquely, apart from displacements as a rigid body. Each three-sided pyramid (tetrahedron) is globally rigid.
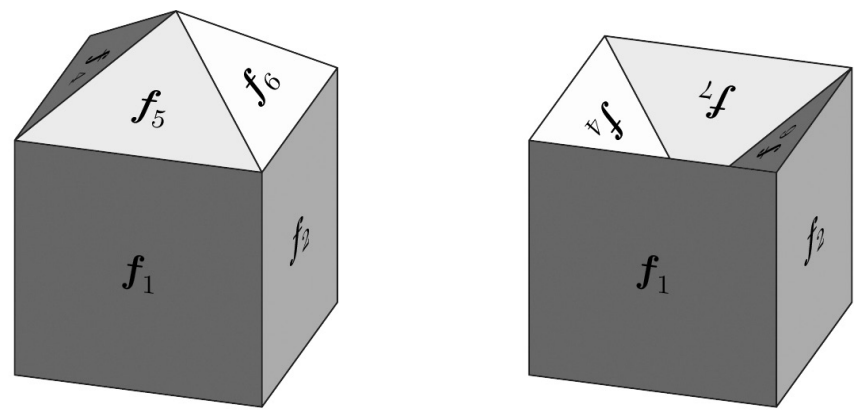
The first important result in the theory of rigidity claims that every convex polyhedron is globally rigid. This is due to A. L. Cauchy, 1813 [3].

A real-world model of a snapping polyhedron might look like a flexible one, but theoretically it is not flexible. The model admits small bendings of the faces and has some clearances at the hinges along the edges, and this causes the seeming flexibility which in any case is somehow limited within a certain neighborhood. A famous example is described in W. Wunderlich's article [18] on a polyhedron exhibited at the science exposition "Phänomena" in 1984 in Zürich. At that time it was falsely stated that this polyhedron is flexible, but it was only snapping between two different snap poses and one spatial shape.

Let us still think of a polyhedron made from cardbord with planar faces, but with variable dihedral angles between any two faces sharing a common edge. A polyhedron is called "continuously flexible", when the dihedral angles of the polyhedron can vary continuously while the intrinsic metric remains invariant. Sometimes, this is called a selfmotion of the structure. R. Bricard classified in 1897 [2] all flexible octahedra, i.e. all flexible four-sided double-pyramids. However, all these polyhedra have self-intersections. Flexible polyhedral structures can be extracted from Bricard's octahedra only when either some faces are omitted [19] or when the polyhedron with 8 triangular faces is seen as a framework with 12 edges.

The first continuously flexible polyhedron without self-intersections was detected in 1977 by R. Connelly [4]. A "flexing sphere" with 9 vertices only was found in 1978 by K. Steffen [13] as a combination of two flexible octahedra.

At the first glance, it might be surprising that even for a regular octahedron there exists a continuously flexible realization with the same intrinsic metric.
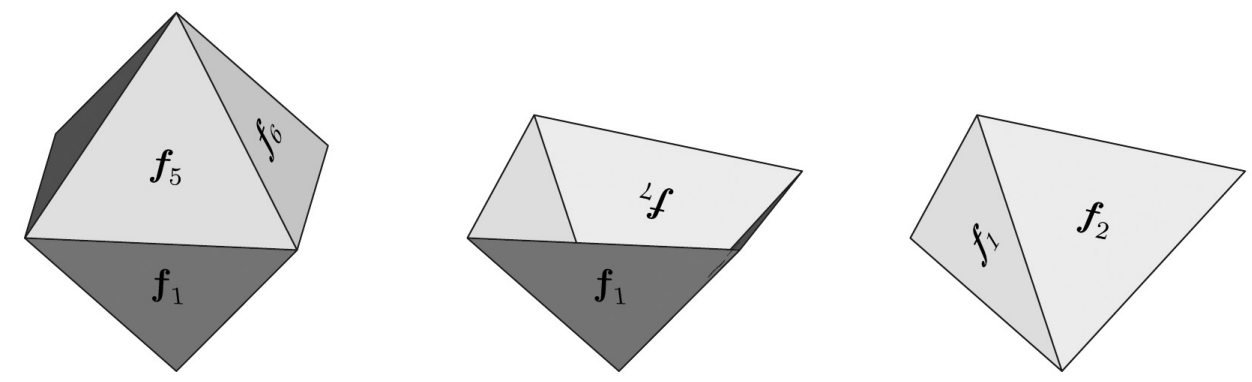
The structure can be re-assembled by putting one four-sided pyramid into the other. This gives a twofold covered quadratic pyramid without basis, which of course is flexible (Fig. 2). This is no contradiction with Cauchy's result, because there we have the restriction to convex spatial shapes only, and the regular octahedron is indeed locally rigid.

It turns out that the computation of the spatial from of any four-sided doublepyramid, i.e., of any general octahedron with given unfolding is an algebraic problem of degree 8 . Hence, up to 8 different realizations are possible. Apart from particular cases, each of these realizations is locally rigid.

The question whether the edge lengths of a polyhedral structure or framework determine its planar or spatial shape uniquely, is also important for many engineering applications, e.g., for mechanical or constructional engineers, for biologists in protein modeling or for the analysis of isomers in chemistry.

In the following text some flexible examples have been presented, and it has been emphasized that there is something between "continuously flexibility" and "rigidity", the "infinitesimal flexibility" which can also be seen as a limiting case of snapping structures after the two different realizations converge. But first of all the terminology and the mathematical background will be clarified.

\section{DEFINITION OF RIGIDITY AND FLEXIBILITY}

In the following definitions polyhedra is not seen not as piecewise linear surfaces, but as frameworks. This means one should concentrate on its edges only. If there are faces with more than three vertices, it must be replaced by some face-to-face tetrahedra erected over this face in order to keep the original face planar. Finally, it should be noted that technical problems like stiffness of edges and clearances along the hinges are not to be of a concern. The focus is just on geometry.

\section{Definition 1 .}

A framework $F$ in $R$ d $, \mathrm{d}=2,3, \ldots$, consists of a set $V=\left\{\mathrm{x}_{1}, \ldots, \mathrm{x}_{v}\right\}$ of vertices and a set $E$ of edges, i.e., $E=\{(i, j) \mid 0<i<j \leq v\}$. The length of the edge $\mathrm{x}_{\mathrm{i}} \mathrm{x}_{\mathrm{j}}$ of $F$ is denoted by $l_{i j}$, and the functions

$$
f_{i j}(\mathrm{y}, \mathrm{z}):=\|\mathrm{y}-\mathrm{z}\|^{2}-l_{\mathrm{ij}}^{2} \text { for } \mathrm{y}, \mathrm{z} \in R^{\mathrm{d}} .
$$

are defined. 
The framework $F$ is called continuously flexible, if there is a continuous family $F_{t}$ of frameworks with vertices $\mathrm{x}_{1}(t), \ldots, \mathrm{x}_{d}(t)$ for $0 \leq t \leq 1$ with $F_{0}=F$ and $f_{i j}$ $\left(\mathrm{x}_{i}(t), \mathrm{x}_{j}(t)\right)=0$ for all $(i, j) \in E$, provided there are at least two vertices $\mathrm{x}_{k}, \mathrm{x}_{l}$ which do not keep their distance constant.

The family $F_{t}, 0 \leq t \leq 1$ is called a flection or self-motion of $F$; each single $F_{t}$ for a fixed $t$ is called a pose of this flection.

It is said that, the edge set $E$ defines the combinatorial structure of $F$. By the request that at least one distance between vertices does not remain constant during the flection, trivial flections are excluded, i.e., pure motions of the framework as a rigid body, expressible in matrix form by

$$
\mathrm{x}_{i}(t)=\mathrm{a}(t)+\mathrm{A}(t) \mathrm{x}_{i} \text { for each } i \mathrm{C}\{1, \ldots, v\}
$$

with a $(t) \in R^{d}$ and an orthogonal $(d \times d)$-matrix a $(t)$, i.e., $\mathrm{A}^{\mathrm{T}}=\mathrm{A}^{-1}$.

The conditions for keeping the lengths of edges constant, are of algebraic nature. Hence, in the case of a continuously flexible $F$ the flection as a function of $t$ is not only continuous but analytic in $t$. Therefore each $\mathrm{x}_{i}(t)$ can be expanded into Taylor series. This is the basis for the following definition.

\section{Definition 2.}

A framework $F$ in $R^{d}$ is called infinitesimally flexible or - more precisely infinitesimally flexible of order $n, n \geq 1$, if for each $i \in\{1, \ldots, v\}$ there is a polynomial function

$$
\mathrm{z}_{i}(t)=\mathrm{x}_{i}+\mathrm{x}_{i, 1} t+\ldots+\mathrm{x}_{i, n^{n}} t^{n}, \mathrm{x}_{i, j} \in R^{d} \text { for } j \in\{1, \ldots, v\}
$$

such that the substitution of $z_{i}(t)$ in the distance functions $f_{i j}$ gives functions with a zero at $t=0$ of multiplicity $>n$, i.e., by using the Landau symbol

$$
f_{i j}\left(\mathrm{z}_{i}(t), \mathrm{z}_{j}(t)\right)=o\left(t^{n}\right) \text { for all }(i, j) \in E,
$$

provided, there is a pair $\left(\mathrm{x}_{k}, \mathrm{x}_{l}\right)$ of vertices with $\left\|\mathrm{z}_{k}-\mathrm{z}_{l}\right\|^{2}-\left\|\mathrm{x}_{k}-\mathrm{x}_{l}\right\|^{2} \neq o\left(t^{n}\right)$. $\mathrm{Z}(t):=\left(\mathrm{z}_{1}(t), \ldots, \mathrm{Z}_{v}(t)\right)$ is called an infinitesimal flection of $F$ of order $n$.

The first derivative $\mathrm{x}_{i, 1}$ of $\mathrm{z}_{i}(t)$ at $t=0$ is called velocity vector. The second derivative $\mathrm{x}_{i, 2}$ in (2) is called acceleration vector of vertex $\mathrm{x}_{i}$.

An infinitesimal flection would be called trivial, if the polynomial functions $z_{i}(t)$ originate from an infinitesimal motion of $F$ as a rigid body, i.e., by an assignment 
$\mathrm{x}_{i, 1}=\mathrm{s}+\mathrm{S} \mathrm{x}_{i}$ with s $\in R[t]^{d}, \mathrm{~S} \in \mathrm{R}[t]^{d \times d}$ and $\mathrm{S}^{\mathrm{T}}=-\mathrm{S}$.

This means, the components of $\mathrm{s}$ and the entries in $\mathrm{S}$ are polynomials in $t$ and matrix $\mathrm{S}$ is skew-symmetric.

\section{Remarks:}

1. In [14] L.S. Velimirović and S.R. Rančić treat the analogue of firstorder flexibility for smooth surfaces. In the flexible case one speaks of infinitesimal bendings of a surface.

2. A framework which admits only trivial flections, is called first order rigid order infinitesimally rigid.

Each continuously flexible framework admits a nontrivial analytic flection and is therefore also infinitesimally flexible of any order. Due to the algebraic character of Eq. (1), for each combinatorial type of framework there is a sufficiently high $n \in N$ such that infinitesimal flexibility of an order $\geq n$ implies continuous flexibility. This was proved by V. Alexandrov in [1]. On the other hand, T. Tarnai presented in frameworks which are infinitesimally flexible of order $2^{m}-1$ for any $m$.

The conditions for a framework of given combinatorial structure to be infinitesimally flexible of given order can be obtained by substituting the polynomial functions $\mathrm{z}_{i}(t)$ in the distance functions $\mathrm{f}_{i j}$ in (1) and comparing the coefficients of all powers of $t$ up to $n$. This results in a series of systems of linear equations. So, checking whether a given framework is rigid or higherorder infinitesimally flexible is reduced to inspecting the solvability of these systems of linear equations step by step.

The converse, i.e., finding the geometric meaning of these conditions, is not as straight forward as one might expect. The system for first order flexibility is homogeneous. Therefore the existence of a nontrivial first-order flection is equivalent to a sufficiently high ranked efficiency of the coefficient matrix, the socalled rigidity matrix of $F$. The solution of the first system defines the values on the right-hand side in the inhomogeneous system for second-order flexibility. When this system is solvable, its solution defines the right-hand side values for the third system, and so on. This more or less technical method has been skipped and the focus in the coming section is on the underlying geometric conditions.

It should be mentioned, that there are several applications of first-order infinitesimal flexibility. In robotics, such infinitesimally flexible poses are 
called singular and usually avoided since at least one degree of freedom is missing there and the control of the robot close to singular poses becomes problematic. When in surveying the relative location of points is determined by measuring some of the mutual distances and when the underlying framework is infinitesimally flexible, then this pose is called critical and results in numerical instability.

\section{INFINITESIMAL FLEXIBILITY VS. SNAPPING FRAMEWORKS}

\section{First order flexibility}

The condition (3) for first-order infinitesimal flexibility means that for each ( $i$, $j) \in E$ in the polynomial $f_{i j}\left(\mathrm{z}_{i}(t), \mathrm{z}_{j}(t)\right)$ the coefficient of $t$ must vanish. This is equivalent to

$$
\left(\mathrm{x}_{i}-\mathrm{x}_{j}\right) \times\left(\mathrm{x}_{i, 1}-\mathrm{x}_{j, 1}\right)=0
$$

This vanishing scalar product means that for each edge $\mathrm{x}_{i} \mathrm{x}_{j}$ of $F$ the components of the velocity vectors of $\mathrm{x}_{i}$ and $\mathrm{x}_{j}$ in direction of the edge are equal. This is called the Projection Theorem (see Fig. 3). To summarize:

\section{Theorem 1.}

A framework $F$ is infinitesimally flexible if to each vertex $\mathrm{x}_{i}$ a velocity vector $\mathrm{x}_{i, 1}$ can be assigned of a type that for all edges of $F$ the Projection Theorem (5) is fulfilled.

The first example in Fig. 4 shows a planar bipartite framework. Bipartite means that the vertices can be subdivided into two sets, and each edge connects

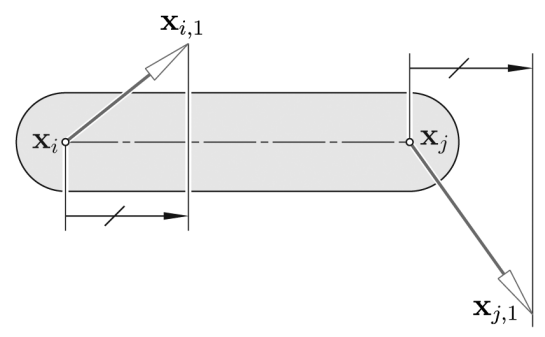

Figure 3 The Projection Theorem (Theorem 1)

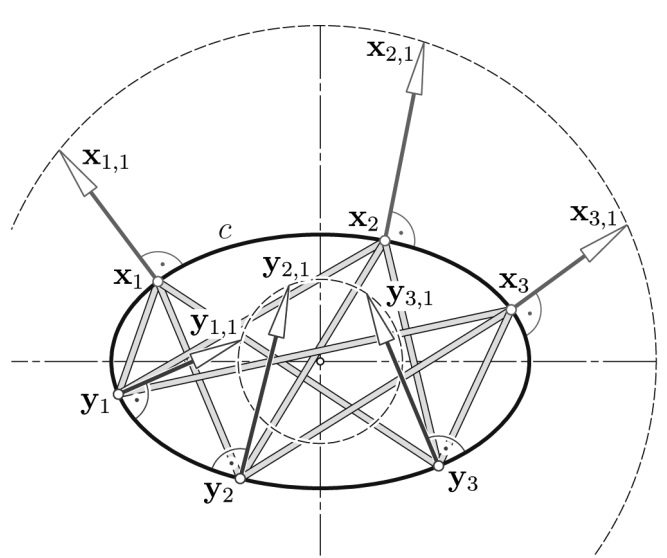

Figure 4 A planar bipartite framework is infinitesimally flexible if and only if the vertices are located on a 2nd-order curve 
points from different sets. In our case there are six vertices $\mathrm{x}_{i}$ and $\mathrm{y}_{j}, i, j \in\{1$, $2,3\}$, and 9 edges $x_{i} y_{i}$. It has been well known at least for one century that this framework is infinitesimally flexible if and only if the vertices are placed on a curve of degree 2, i.e., either on a conic $c$ or on two lines. This is still true when more than 6 points $\mathrm{x}_{i}$ and $\mathrm{y}_{j}$ are specified on the same conic.

The analogous result is valid for any dimension $\mathrm{d}$ when the conic is replaced by any quadric in $R^{d}$. The following short proof owing to W. Whiteley [16] reveals that this condition is sufficient and that the velocity vectors can be chosen perpendicular to the quadric, as shown for $d=2$ in Fig. 4.

\section{Proof:}

The coordinate vectors are written in columns and the equation of the quadric is set up in matrix form by $\mathrm{x}^{\mathrm{T}} \mathrm{Qx}=k$ with a symmetric $(d \times d)$-matrix $\mathrm{Q}$. Since $\mathrm{x}_{i}$ and $\mathrm{y}_{j}$ are located on the quadric, we have $\mathrm{x}_{i}^{\mathrm{T}} \mathrm{Q} \mathrm{x}_{i}=\mathrm{y}_{j}{ }^{\mathrm{T}} \mathrm{Qy} \mathrm{y}_{j}=k$. Now the velocity vectors are specified by $\mathrm{x}_{i, 1}=\mathrm{Qx}_{i}$ and $\mathrm{y}_{i, 1}=-\mathrm{Qy}_{i}$, and for the edge $\mathrm{x}_{i} \mathrm{y}_{j}$ it is verified that the Projection Theorem has been fulfilled. For this purpose the scalar product is written in matrix form and the following obtained

$$
\begin{aligned}
& \left(\mathrm{x}_{i}-\mathrm{y}_{j}\right)^{\mathrm{T}}\left(\mathrm{x}_{i, 1}-\mathrm{y}_{j, 1}\right)=\left(\mathrm{x}_{i}-\mathrm{y}_{j}\right)^{\mathrm{T}}\left(\mathrm{Qx}_{i}+\mathrm{Qy}\right) \\
& =\mathrm{x}_{i}^{\mathrm{T}} \mathrm{Qx}_{i}-\mathrm{y}_{j}^{\mathrm{T}} \mathrm{Qx}_{i}+\mathrm{x}_{i}^{\mathrm{T}} \mathrm{Qy}_{j}-\mathrm{y}_{j}^{\mathrm{T}} \mathrm{Qy}_{j}=0,
\end{aligned}
$$

since $\mathrm{x}_{i}$ and $\mathrm{y}_{j}$ fulfill the quadric's equation and the real number $\mathrm{x}_{i}^{\mathrm{T}} \mathrm{Qy_{j }}$ equals its transpose $\mathrm{y}_{j}^{\mathrm{T}} \mathrm{Q}^{\mathrm{T}} \mathrm{x}_{i}=\mathrm{y}_{j}^{\mathrm{T}} \mathrm{Qx_{i }}$.

On the other hand, $\left(\mathrm{x}_{i}^{\mathrm{T}} \mathrm{Q}\right) \mathrm{x}=0$ is the equation of the tangent plane of the quadric at point $\mathrm{x}_{i}$, and hence the gradient of this plane, the vector $\mathrm{x}_{i, 1}=\mathrm{Qx}_{i}$ is perpendicular to the quadric. The same holds true for point $\mathrm{y}_{j}$ (Fig. 4).
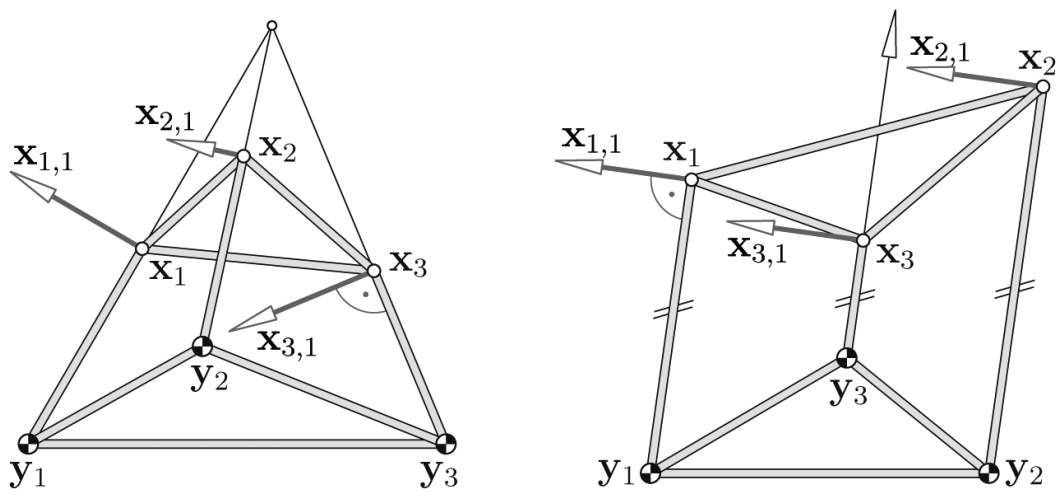
The assignment of velocity vectors $\mathrm{x}_{i, 1}$ to the vertices $\mathrm{x}_{i}$ of an infinitesimally flexible framework $F$ is not unique. Apart from a scaling, i.e., replacement of $\mathrm{x}_{i, 1}$ by $\alpha \mathrm{x}_{i, 1}$ for any fixed $\alpha \in R \backslash\{0\}$, an instantaneous motion can additionally be imposed according to (4). This means that to each $\mathrm{x}_{i, 1}$ the vector $\mathrm{s}+\mathrm{Sx}_{i}$ with $\mathrm{S}^{\mathrm{T}}=-\mathrm{S}$ can be added without disturbing Eq. (5) since $\left(\mathrm{x}_{i}-\mathrm{x}_{j}\right)^{\mathrm{T}} \mathrm{S}\left(\mathrm{x}_{i}-\mathrm{x}_{j}\right)$ is the null form.

The next example displayed in Fig. 5 is again a planar framework with 6 vertices and 9 edges, but not bipartite. It is a pinned framework, i.e., vertices indicated by the black-and-white points in Fig. 5 are fixed. This framework is infinitesimally flexible if and only if the three lines $x_{i} y_{i}$ have one point in common or are parallel.

In the examples presented up to now (Figs. 4 and 5) the geometric characterization of infinitesimally flexible frameworks is of projective nature. If a collineation is applied on the flexible framework, it still remains infinitesimally flexible. This is surprising since rigidity is based on metric properties and they are changed under collinear transformations. However, the projective invariance of infinitesimal flexibility is a classical result and probably first proved in 1920 by H. Liebmann (1920) [7]. Alternative proofs can be found in [15] and [5].

Only first-order infinitesimal flexibility is projectively invariant. This follows from the examples of higher-order flexible frameworks presented in [8] and [9].

Infinitesimal flexibility can be seen as the limiting case where two realizations of a framework coincide. This was the way how W. Wunderlich studied infinitesimal flexibility. The next theorem reveals that there is a direct connection between snapping frameworks and infinitesimally flexible frameworks of the same combinatorial type. W. Whiteley [17] calls this correspondence "averaging"; in I. Izmestiev's paper [5] it is called Pogorelov map.

\section{Theorem 2.}

Let $\mathrm{y}_{1}, \ldots, \mathrm{y}_{v}$ and $\mathrm{y}_{1}^{\prime}, \ldots, \mathrm{y}^{\prime}{ }_{v}$ be the vertices of two incongruent realizations of a framework $F$ with the same intrinsic metric, i.e., with the same edge lengths $l_{i j}$. Then the midpoints $\mathrm{x}_{i}=1 / 2\left(\mathrm{y}_{i}+\mathrm{y}_{i}^{\prime}\right)$ of corresponding vertices make a framework $F$ of the same combinatorial structure which is infinitesimally flexible with velocity vectors $\mathrm{x}_{i, 1}=1 / 2\left(\mathrm{y}_{i}-\mathrm{y}_{i}^{\prime}\right)$.

Conversely, any infinitesimally flexible framework $F$ with vertices $\mathrm{x}^{1}, \ldots, \mathrm{x}_{v}$ and velocity vectors $\mathrm{x}_{1,1}, \ldots, \mathrm{x}_{v, 1}$ gives rise to two incongruent realizations of a 
framework $F$ of the same combinatorial type, namely that with vertices $\mathrm{y}_{i}=\mathrm{x}_{i}$ $+\mathrm{x}_{i, 1}$ and $\mathrm{y}_{i}^{\prime}=\mathrm{x}_{i}-\mathrm{x}_{i, 1}$, respectively.

\section{Proof:}

The proof is unexpectedly short. For each edge of $F$, i.e., for each $(i, j) \in E$ the equations $\left\|\mathrm{y}_{i}-\mathrm{y}_{j}\right\|=\left\|\mathrm{y}_{i}^{\prime}-\mathrm{y}_{j}^{\prime}\right\|$ can be rewritten as

$$
\left(\mathrm{y}_{i}-\mathrm{y}_{j}\right)^{2}-\left(\mathrm{y}_{i}^{\prime}-\mathrm{y}_{j}^{\prime}\right)^{2}=0
$$

which is equivalent to

or

$$
\left(\mathrm{y}_{i}-\mathrm{y}_{j}+\mathrm{y}_{i}^{\prime}-\mathrm{y}_{j}^{\prime}\right) \times\left(\mathrm{y}_{i}-\mathrm{y}_{j}-\mathrm{y}_{i}^{\prime}+\mathrm{y}_{j}^{\prime}\right)=0
$$

$$
\left(\left(\mathrm{y}_{i}+\mathrm{y}_{i}^{\prime}\right)-\left(\mathrm{y}_{j}+\mathrm{y}_{j}^{\prime}\right)\right) \times\left(\left(\mathrm{y}_{i}-\mathrm{y}_{i}^{\prime}\right)-\left(\mathrm{y}_{j}-\mathrm{y}_{j}^{\prime}\right)\right)=0 .
$$

This is just the statement of the Projection Theorem (5) $\left(\mathrm{x}_{i}-\mathrm{x}_{j}\right) \times\left(\mathrm{x}_{i, 1}-\mathrm{x}_{j, 1}\right)=0$ because of $2 \mathrm{x}_{i}=\mathrm{y}_{i}+\mathrm{y}_{i}^{\prime}$ and $2 \mathrm{x}_{i, 1}=\mathrm{y}_{i}-\mathrm{y}_{i}^{\prime}$.

For any given infinitesimally flexible framework $F$ the appointed velocity vectors $\mathrm{x}_{i, 1}$ can be replaced simultaneously by $\alpha \mathrm{x}_{i, 1}$ for any $\alpha \in R$. The smaller the absolute value of $\alpha$, the closer the two obtained snapping poses are. The given flexible framework $F$ is the limit for $\alpha \rightarrow 0$.

Figure 6 shows on the left-hand side two realizations derived from the infinitesimally flexible framework in Fig. 5, left. On the right-hand side it is illustrated how from the two realizations by the principle of "averaging" the infinitesimally flexible $F$ is obtained. It can be proved that displacements of $F^{\prime}$ relative to $F$ do not change the shape of $F$.
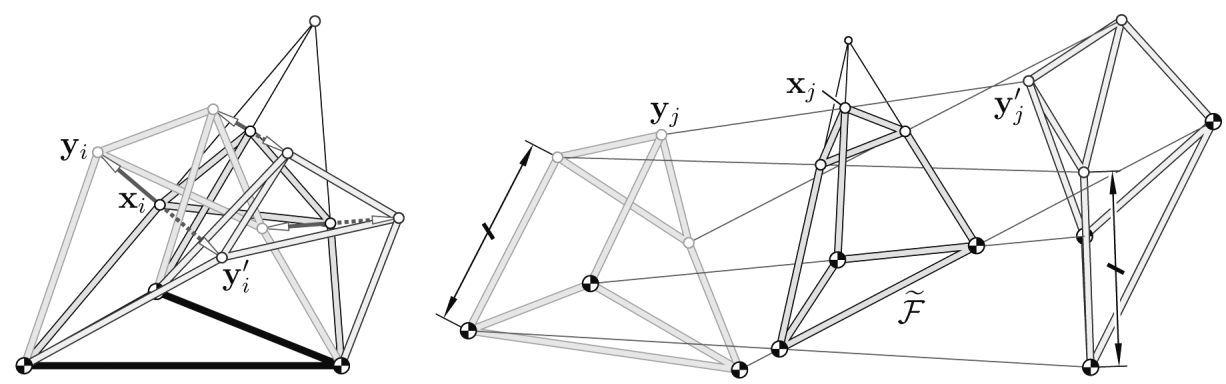
It could be proceeded in the same way with the infinitesimally bipartite framework obtained from Fig. 4. According to [10] it is known, that after a suitable displacement of one of the two realizations the vertices of any snapping bipartite framework are located on two confocal conics (quadrics). Then the snapping is the result of interchanging the two conics.

\section{Flexible polygonal structures}

The conclusion is with polyhedral structures which play a role in paper folding (origami) but also in new architectural surface design as quad meshes. The starting point is with a Kokotsakis mesh (German: Vierflach), an object named after A. Kokotsakis [6]. A quadrangular Kokotsakis mesh is the compound of $3 \times 3$ planar quadrangles. In Fig. 7, left, the scheme of a quadrangular Kokotsakis mesh is shown with a central face $f_{0}$ and a belt of 8 quadrangles around it. On the right hand side a flection of a continuously flexible version is displayed.

Though a complete classification of all flexible cases is still an open problem, some particular conditions are known which give rise to continuously flexible meshes (compare, e.g., [12]).

The start will be with the infinitesimally flexible case. The geometric characterization of these meshes is already given in [6] (see Fig. 4). Kokotsakis' arguments have been followed which are based on standard results from Kinematics. For any two faces $f_{i}, f_{j}$ sharing an edge, the edge is the axis $i j$ of the relative motion. According to the Three-Pole-Theorem for any three faces $f_{i}$, $f_{j}, f_{k}$ with rotations as pairwise relative motions the three axes $i j, i k$ and $j k$ must
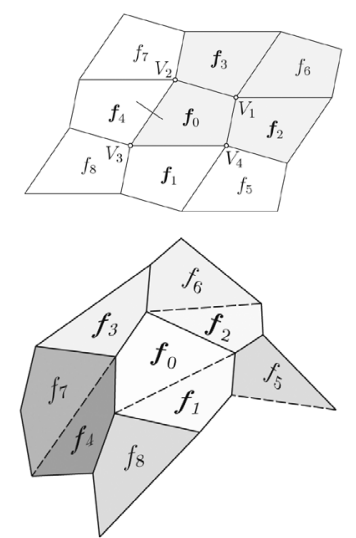

Figure 7

Up: Scheme of a Kokotsakis mesh.

Down: Pose of a continuously flexible Kokotsakis mesh with central face $\mathrm{f}_{0}$; dashes indicate valley folds.
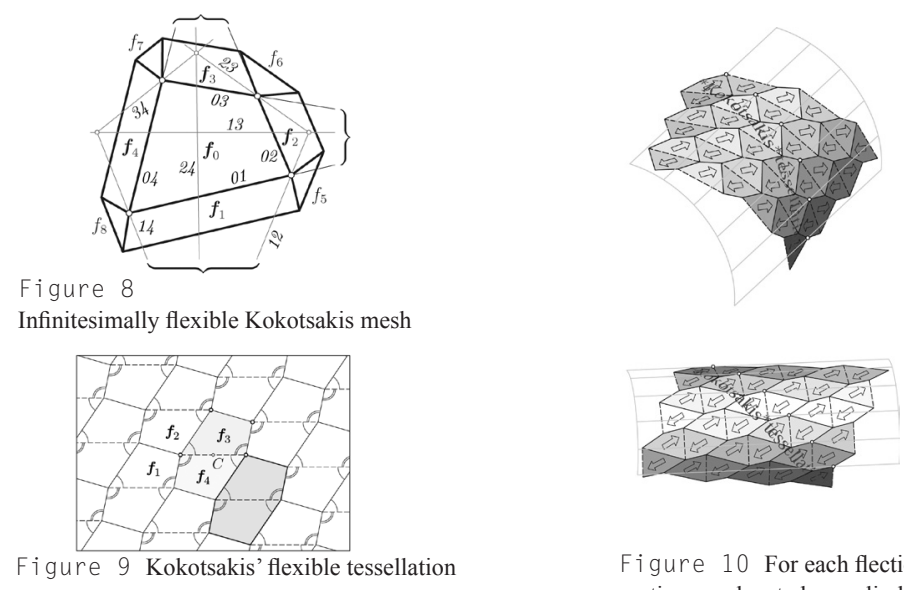

Figure 10 For each flection the vertices are located on cylinders of rotation 
be coplanar and share a point. This implies, e.g., that at the Kokotsakis mesh (see Fig. 8) the axis 12 of the relative motion between $f_{1}$ and $f_{2}$ is the line of intersection between the planes spanned by $f_{5}$ and $f_{0}$. The lines of intersection with the plane are called of $f_{0}$ traces. In Fig. 8 the mesh is cut by a plane parallel to that of $f_{0}$.

\section{Theorem 3.}

A Kokotsakis mesh is infinitesimally flexible if and only if the following three points are collinear, the points of intersection between the traces of $\left(f_{1}, f_{3}\right),\left(f_{5}\right.$, $\left.f_{6}\right)$ and $\left(f_{7}, f_{8}\right)$. This is equivalent to the statement that the points of intersection between the traces of $\left(f_{2}, f_{4}\right),\left(f_{6}, f_{7}\right)$ and $\left(f_{8}, f_{5}\right)$ are aligned.

By the way, the equivalence between the two "collinearities" is just a consequence of Desargues' theorem.

According to Theorem 2 each infinitesimally flexible case gives rise to pairs of snapping Kokotsakis meshes. There are even examples where one realization is flat.

An interesting continuously flexible quad mesh, a polyhedral compound of $m \times n$ planar quadrangles, dates also back to Kokotsakis [6]. One starts with a planar tessellation by congruent non-convex quadrangles (Fig. 9) with the property, that any two quadrangles sharing a side can be interchanged by a $180^{\circ}$-rotation about the midpoint $C$ of the common side. These two adjacent quadrangles form a centrally symmetric hexagon, and the tessellation can also be generated by translations of this hexagon.

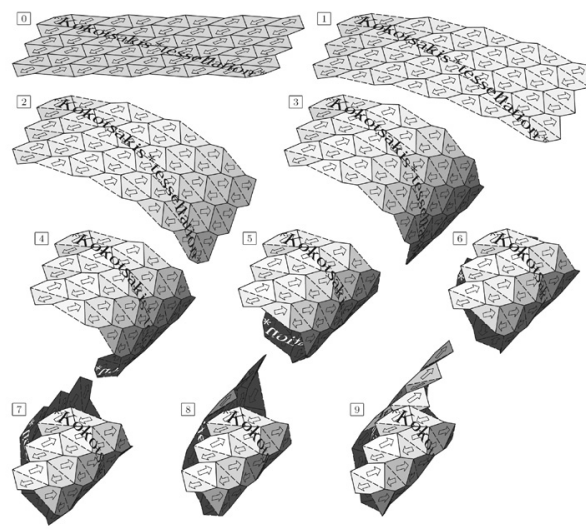

Figure 11 Flection of the Kokotsakis tesselation of the first kind

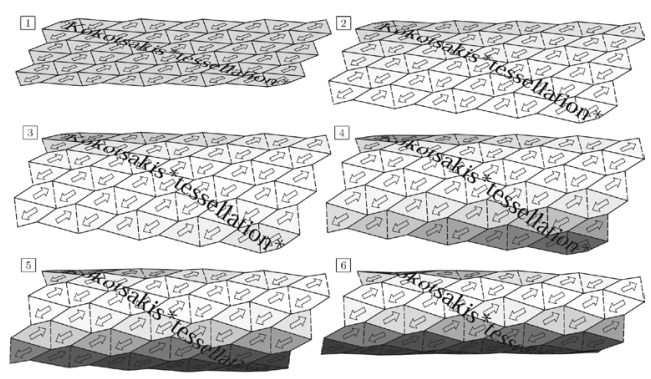


Any connected portion of this tessellation is continuously flexible. As explained in [11], the flexibility can be proved by starting with a four-sided pyramid of quadrangles sharing a vertex and by continuing this flection step by step to the complete tesselation.

It turns out that starting from the flat initial pose, there are two differentiable types of bendings of this piecewise linear surface. In each non-flat realization all vertices are located on a cylinder of revolution (see Fig. 10). Hence the polygonal structure of each realization gives a discrete approximation of this cylinder.

Figures 11 and 12 show snapshots of these two bendings. The edges of the planar tessellation can be combined to two folds. And for each fold every second vertex lies on the same helical line. When the basic quadrangle is a trapezoid, then the folds of one family become aligned, and one family of bendings is that of a prism and therefore trivial. 113-148 (1897). (1813). 140, 183-203 (2009).

$6 \quad$ Kokotsakis, A., Über bewegliche Polyeder, Math. Ann. 107, 627-647 (1932).

Liebmann, H., Ausnahmefachwerke und ihre Determinante, Sb. Bayer. Akad.Wiss. 1920, 197-227. 
Stachel, H., Higher-Order Flexibility for a Bipartite Planar Framework, In A. Kecskem'ethy, M. Schneider, C. Woernle (eds.): Advances in Multi-body Systems and Mechatronics. Inst. f. Mechanik und Getriebelehre, TU Graz, Duisburg 1999, 345-357.

Stachel, H., Configuration Theorems on Bipartite Frameworks, Rend. Circ. Mat. Palermo, II. Ser., 70, 335-351 (2002).

Stachel, H., Remarks on Miura-ori, a Japanese Folding Method. Acta Technica Napocensis, Ser. Applied Mathematics and Mechanics 52, Vol. Ia, 245-248 (2009).

Stachel, H., A kinematic approach to Kokotsakis meshes, Comput. Aided Geom. Des. (to appear).

Steffen, K.: A symmetric flexible Connelly sphere with only nine vertices. http://www.math. cornell.edu/ connelly/Steffen.pdf

Velimirović, L.S., Rančić, S.R.: Notes on Infinitesimal Bending of a Toroid Formed by Revolution of a Polygonal Meridian. J. Geometry Graphics 13, no. 2, 177-186 (2009).

Wegner, B., "On the projective invariance of shaky structures in Euclidean space", Acta Mech. 53, 163-171 (1984).

Whiteley, W., Infinitesimal motions of a bipartite framework, Pacific J. of Math. 110, 233-255 (1984).

Whiteley, W., "Rigidity and scene analysis". In J.E. Goodman, J. O’Rourke (eds.): Handbook of Discrete and Computational Geometry, CRC Press, Boca Raton, New York 1997.

Wunderlich, W., Schwabe, C.: Eine Familie von geschlossenen gleichfl"achigen Polyedern, die fast beweglich sind. Elem. Math. 41 (1986), 88-98.

Wunderlich, W.: Starre, kippende, wackelige und bewegliche Achtflache. Elem. Math. 20 (1965), 25-32.

N.B. This research is supported by Grant No. I 408-N13 of the Austrian Science Fund FWF within the project "Flexible polyhedra and frameworks in different spaces", an international cooperation between FWF and RFBR, the Russian Foundation for Basic Research. 